\title{
High Accuracy and Conservative Numerical Model forTreatment of Advection Terms
}

\author{
RuiJin Zhang ${ }^{1,2, a}$, XueZhi Huang $^{1}$ and JinMei Zhou ${ }^{1}$ \\ ${ }^{1}$ College of Marine Environmental Eng., Dalian Ocean Univ., Dalian 116023, China \\ ${ }^{2}$ Key Laboratory of Nearshore Marine Environmental Research, Dalian 116023,China \\ aruijinz@dlou.edu.cn
}

Keywords: Advection Terms,CIP,CIP-CLS2,CIPCLSR1

\begin{abstract}
The difficulty in solving the shallow water equation lies in treating the advection terms. Therefore numerical simulation of advection term is the key to the numerical simulation of shallow water equation. In this paper, original CIP method, CIP-CSL2 method, CIP-CLSR1 method and first-order unwind scheme are adopted to treat the advection term.
\end{abstract}

\section{Introduction}

The original cubic-interpolated propagation (CIP) method, developed by Yabe and co-workers ${ }^{[1-2]}$ is such a method that is suitable to solve the advection terms. The CIP scheme is a low numerical diffusion and stable scheme, and can solve hyperbolic equations with three-order accuracy in space and has been successfully applied to various complex fluid flow problems.

It is frequently demonstrated that the CIP method shows good conservation of mass, although the method is written in a non-conservative form. But in a special case such as the oceanic problems, exact conservation is needed. In such situation, new conservative semi-Lagrangian schemes called CIP-CLS have been successfully developed, such as CIP-CSL4, CIP-CLS2 ${ }^{[3]}$,CIP-CLS3. In order to avoid the oscillation in the calculation, rational function is added to the CIP-CSL2 method, which are called as CIP-CLSR $1^{[4]}$ and CIP-CLSR2. The schemes are based on the concept of the CIP schemes and preserve the excellent numerical features of the CIP scheme. The schemes are written as semi-Lagrangian formulations and provide stable solutions under large CFL with exact mass conservation. Since these schemes do not use the cubic polynomial but uses different orders of polynomials, the name of these CIP families are refined as constraint interpolation profiles and still keep the abbreviation CIP.

\section{Simple Review of CIP, CIP-CLS2 and CIPCLSR1 methods}

Strategy of CIP method

Here we explain the strategy of CIP method by using a one-dimensional advection equation,

$$
\frac{\partial f}{\partial t}+u \frac{\partial f}{\partial x}=0
$$

When the velocity is constant, the solution of Eq. 1 gives a simple translational motion of field $f$ with a velocity of $u$.

CIP-CLS2 method

In this section, a method to solve the one-dimensional conservative equation is described.

$$
\frac{\partial f}{\partial t}+\frac{\partial(u f)}{\partial x}=0
$$

where $u$ is a variable now.

the CIP-CSL2 requires an additional constraint of the value integrated over neighboring two points, 


$$
\rho_{i}^{n}=\int_{x_{i}}^{x_{l+1}} f(x, t) d x
$$

where $n$ indicates the time .

\section{CIP-CLSR1 method}

Here consider the advection transport equation in a conservative form again,

$$
\frac{\partial f}{\partial t}+\frac{\partial(u f)}{\partial x}=0
$$

A numerical scheme can be constructed using directly a so-called flux form, like

$$
\frac{\partial \rho_{i}}{\partial t}=-\frac{(u f)_{i+\frac{1}{2}}-(u f)_{i-\frac{1}{2}}}{\Delta x_{i}}
$$

where $\Delta x_{i}=x_{i+1 / 2}-x_{i-1 / 2}$. Here $\rho_{\imath}$ denotes the cell-integrated average of the transported quantity, while $\left(u f_{i+1 / 2}\right.$ and $(u f)_{i-1 / 2}$ the fluxes across the cell boundaries. The resulting numerical formulation is in general automatically conservative.

\section{Verification of the CIP series methods}

Before applying these methods to solve the shallow water equations, it is necessary to verify their accuracy and conservation

Verification of one-dimensional methods

A one-dimensional square wave advected with a constant velocity field was computed with different spatial solutions: first order upwind scheme, CIP method, CIPCSL2 method and CIPCLSR1 method.

The initial condition is:

$$
f(o, x)= \begin{cases}1 & \text { if } 0.7 \leq x \leq 1.3 \\ 0 & \text { otherwise }\end{cases}
$$

The CFL number for this calculation is 0.1 .

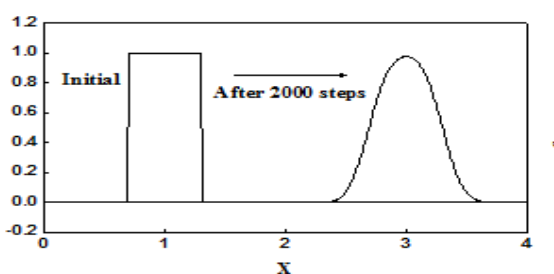

(a) First order upwind scheme

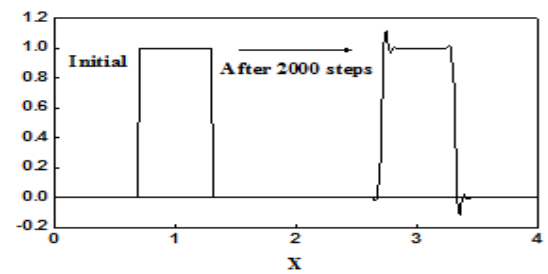

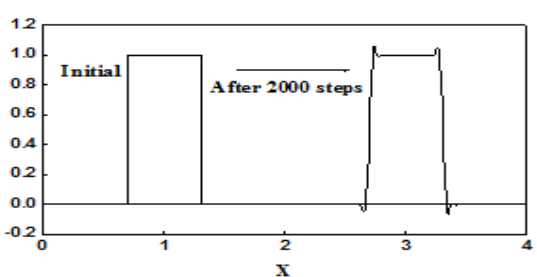

(b) CIP method

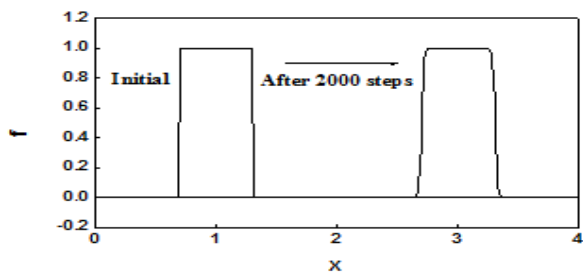

Fig. 1 Comparison of one-dimensional advected square wave with a constant velocity field by different spatial solutions

From Fig. 1, it can be seen that in the calculation with first order upwind scheme, the shape of the advected quantity the wave is quite different with the original one and has the biggest numerical errors in the four methods, due to the numerical diffusion of first order upwind scheme. On the other hand, this numerical diffusion makes the calculation stable.

All the three CIP methods can preserve the shape of the advected quantity well and give small numerical errors. CIPCSL2 method and CIPCSLR1 method gives very similar numerical errors, while CIP method gives relatively smaller numerical errors. However numerical oscillations near discontinuity can be found in CIP method and CIPCSL2 method.

Verification of two-dimensional methods

To compare the properties of these methods, numerical errors after 1 revolution and 2 revolutions 
comparison is shown in Table 1.

Table 1 Comparison of the numerical errors by different spatial solution

\begin{tabular}{ccccc}
\hline Scheme & 1st order Upwind & CIP & CIPCSL2 & CIPCLSR1 \\
\hline $\mathrm{E}_{\text {TОт }}$ (1 revolution) & $2.694 \mathrm{E}-02$ & $1.0727 \mathrm{E}-02$ & $2.876 \mathrm{E}-03$ & $3.157 \mathrm{E}-03$ \\
\hline $\mathrm{E}_{\mathrm{TOT}}$ (2 revolutions) & $4.069 \mathrm{E}-02$ & $1.7384 \mathrm{E}-02$ & $3.430 \mathrm{E}-03$ & $3.780 \mathrm{E}-03$ \\
\hline
\end{tabular}

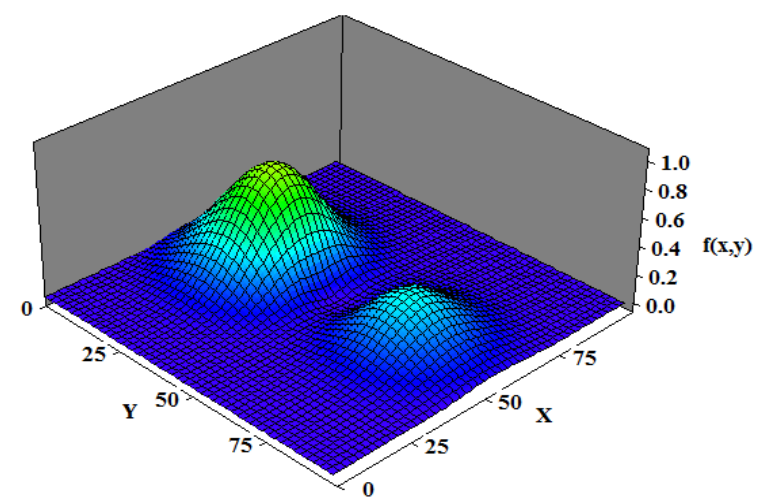

Fig. 2 Numerical solution after 1 revolution of Molenkamp-Crowley test by: $1^{\text {st }}$ upwind schem

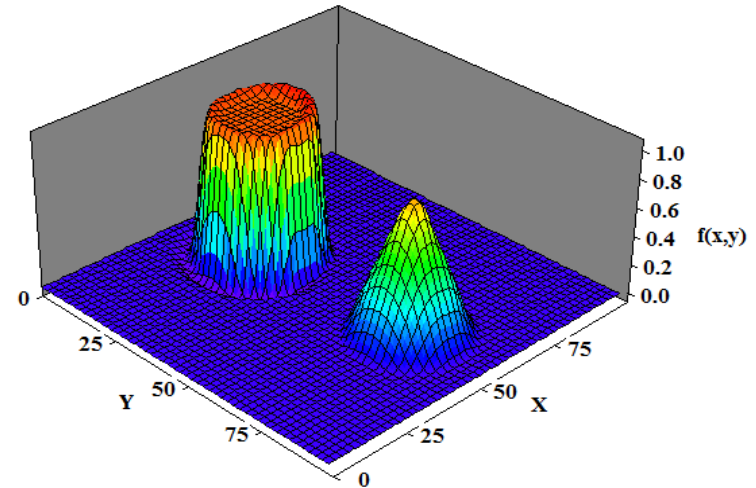

Fig. 4 Numerical solution after 1 revolution of Molenkamp-Crowley test by: CIP-CSL2

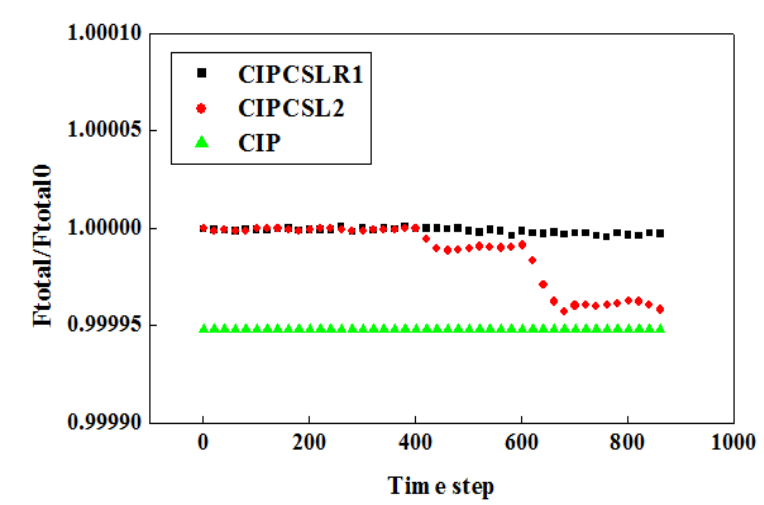

Fig. 6 CIPCSLR1,CIPCSL2 and CIP method

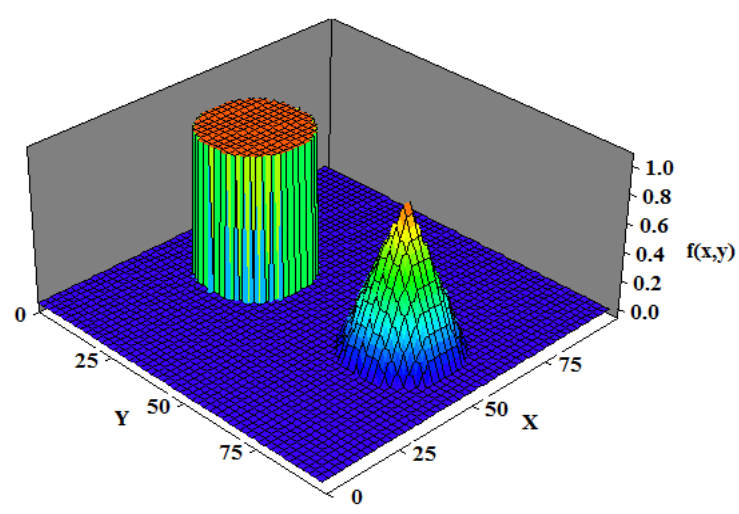

Fig. 3 Numerical solution after 1 revolution of Molenkamp-Crowley test by: CIP method

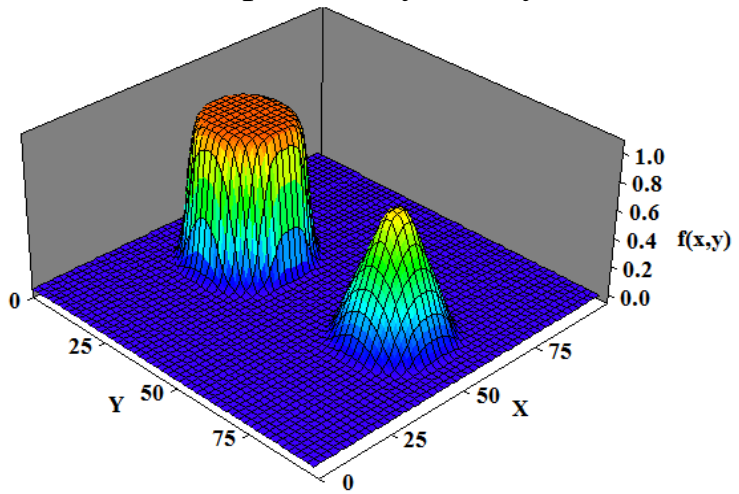

Fig. 5 Numerical solution after 1 revolution of Molenkamp-Crowley test by: CIP-CSLR1

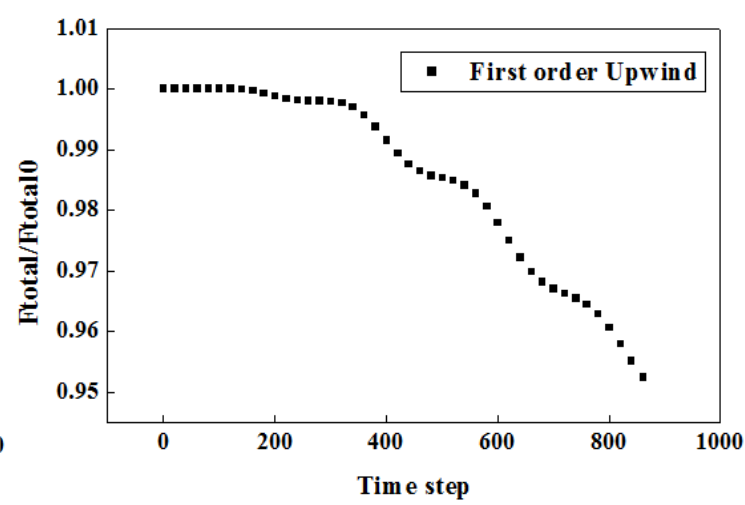

Fig. 7 First order upwind scheme

2D Molenkamp-Crowley test problem ${ }^{[5]}$ was computed with different spatial solutions: first order upwind scheme, CIP method, CIPCSL2 method and CIPCLSR1 method.

It can be inferred from Fig. 2 to Fig. 7, due to the numerical diffusion of first order upwind scheme, the shape of the cylinder and the cone is quite different with the original one and has the biggest numerical errors in the four methods and has the worst conservation.

All the three CIP methods can preserve the shape of the advected quantity well and give small numerical errors. And CIP method gives the best results with the smallest numerical errors in the 
four methods. CIPCSLR1 method gives the best conservation.

\section{Summary}

Numerical model based on original CIP method, CIP-CSL2 and CIP-CSLR1 method are developed numerical comparison of these three CIP methods and first-order upwind scheme have been performed. These three CIP methods show the high accuracy and conservation, among which, CIP-CSLR1 method is also oscillation-less. These three CIP methods are suitable to treat the advection terms.

\section{Acknowledgment}

Thanks for the support of the National Natural Science Foundation of China (No.31302232)

\section{References}

[1]Takewaki, H., Nishiguchi, A. and Yabe, T.. The cubic interpolation pseudo -particle method (CIP) for solving hyperbolic-type equations. Journal of computational physics, Vol.61, pp.261-268, 1985.

[2]Yabe T. and Aoki T.. A universal solver hyperbolic-equations by cubic-polynomial interpolation. I. One-dimensional solver. Computer Physics Communications, Vol.66, pp.219-232, 1991.

[3]Yabe T., Tanaka R., Nakamura T. and Xiao F.. An exactly conservative semi-Lagrangian scheme (CIP-CSL) in one dimension. Monthly Weather Review, Vol.129, pp.332-344, 2001.

[4]Xiao F., Yabe T., Peng X. and Kobayashi H.. Conservative and oscillation-less atmospheric transport schemes based on rational functions [J]. Journal of Geophysical Research, Vol.107, D22, 4609, doi: 10.1029/2001JD001532: ACL 2-1-2-11, 2002.

[5]Verwer, J. G., W. H. Hundsdorfer and J. G. Bolm. Numerical time integration for air pollution models. CWI Report, MAS-R9825,1988. 